\title{
Is there a role for sequential afatinib and osimertinib in patients with EGFR mutation?
}

\author{
Natsuhiko Iwamoto $^{1}$, Young Hak Kim ${ }^{1}$, Shigeaki Iwatsubo ${ }^{1}$, Yoshihiro Nishimura ${ }^{2}$, Yasuhiro Funada ${ }^{1}$ \\ ${ }^{1}$ Department of Respiratory Medicine, Takatsuki General Hospital, Takatsuki, Osaka, Japan; ${ }^{2}$ Department of Respiratory Medicine, Graduate School \\ of Medicine, Kobe University, Kobe, Japan \\ Correspondence to: Young Hak Kim, MD, PhD. Department of Respiratory Medicine, Takatsuki General Hospital, 1-3-13 Kosobe-cho, Takatsuki, \\ Osaka 569-1192, Japan. Email: ykim.free@gmail.com.
}

Submitted Oct 27, 2021. Accepted for publication Dec 30, 2021.

doi: $10.21037 /$ jtd-21-1700

View this article at: https://dx.doi.org/10.21037/jtd-21-1700

Epidermal growth factor receptor (EGFR) mutation is the most common driver mutation in patients with non-small cell lung cancer (NSCLC), and multiple tyrosine kinase inhibitors (TKIs) have been developed targeting EGFR. Accordingly, there are several treatment approaches for this patient population. Recently, Popat et al. presented the results of the UpSwingG study, a global observational study of patients with NSCLC and EGFR mutation who received sequential afatinib and osimertinib treatment (1). The primary objective of the study was to evaluate time to treatment failure (TTF), i.e., the time from the first dose of afatinib to the last dose of osimertinib or death by any cause, and the key secondary objectives were overall survival (OS) and objective response rate (ORR). The median TTF and OS were 27.7 months [95\% confidence interval (CI): 24.0-30.2] and 36.5 months (95\% CI: 32.9-41.8), respectively, and the ORR was $74 \%$ for afatinib and $45 \%$ for osimertinib. The authors concluded that sequential afatinib and osimertinib may be a reasonable treatment option for patients with NSCLC and EGFR mutation, and similar results have been reported previously in the GioTag study (2). However, both the UpSwingG and GioTag studies are observational studies that only included T790M mutation-positive patients who most benefit from osimertinib after afatinib failure. As the authors of both studies mentioned, this is the best scenario for EGFR mutation-positive NSCLC patients receiving afatinib as their initial treatment.

The current standard first-line treatment for NSCLC patients with $E G F R$ mutation is osimertinib. In the FLAURA study, the median OS for osimertinib was
38.6 months (95\% CI: $34.5-41.8$ ), which is numerically similar to that found in the UpSwingG and GioTag studies (1-3). However, a subgroup analysis of the FLAURA study revealed that there was no significant OS difference in patients with L858R mutation, while those with ex 19del derived significant OS benefit from osimertinib (3). Considering its significant OS benefit, mild toxicity, and no need for re-biopsy, osimertinib seems to be the best choice for patients with ex 19del. However, more effective treatment is necessary for patients with L858R mutation.

Looking at the efficacy of sequential afatinib and osimertinib treatment based on the mutational subtypes, the median OS was inferior in patients with L858R mutation in both the UpSwingG study (38.0 months for ex 19del $v$ s. 33.1 months for L858R mutation) (1) and GioTag study (41.6 months for ex 19del vs. 33.0 months for L858R mutation) (2). In addition, patients with L858R mutation are less likely to develop T790M after receiving first- or second-generation EGFR-TKI. According to two Japanese studies, the prevalence of T790M after receiving first- or second-generation EGFR-TKI is $55.6 \%$ and $63.4 \%$ for patients with ex $19 \mathrm{del}$ and $43.0 \%$ and $37.5 \%$ for patients with L858R mutation, respectively $(4,5)$. In fact, the distributions of ex 19del and L858R mutation were 70.7\% and $29.3 \%$ in the UpSwingG study and $73.5 \%$ and $26.0 \%$ in the GioTag study, respectively. To summarize, patients with L858R mutation have less chance to develop T790M after afatinib failure, and, more importantly, the treatment efficacy of sequential afatinib and osimertinib for L858R mutation is inferior to that for ex $19 \mathrm{del}$.

In this respect, combination therapies with EGFR- 
TKI and vascular endothelial growth factor (VEGF) or VEGF-receptor (VEGFR) inhibitors are promising. In the RELAY study, although the OS data are still immature, progression-free survival was significantly better for the erlotinib+ramucirumab arm of ex $19 \mathrm{del}$ [hazard ratio (HR) 0.65; $\mathrm{P}=0.0098$ ] and L858R mutation (HR 0.62; $\mathrm{P}=0.0060)$ (6). There is growing evidence that compound $E G F R$ mutations and concomitant mutations outside of the EGFR gene, which are significantly associated with the reduced efficacy of EGFR-TKI, are more prevalent in patients with L858R mutation compared to patients with ex 19del (7). Adding a VEGF(R) inhibitor to EGFR-TKI may improve the outcomes of patients with L858R mutation compared with EGFR-TKI alone. The NEJ026 study failed to demonstrate significant OS improvement with erlotinib+bevacizumab in patients with $\mathrm{L} 858 \mathrm{R}$ mutation (HR 0.79: 95\% CI: 0.460-1.358); however, the study was not designed to detect statistical OS difference in each mutational subtype. Furthermore, effective subsequent treatment might have compromised statistically significant PFS improvement of erlotinib+bevacizumab (8).

In conclusion, for patients with ex 19del, osimertinib is currently the best treatment option, though the results of an ongoing comparative study are still awaited (9). On the other hand, more effective treatment is necessary for patients with L858R mutation, and combination with EGFR-TKI and a VEGF(R) inhibitor seems most effective for this patient population. Currently, several phase III studies comparing osimertinib and osimertinib + VEGF(R) inhibitor are ongoing $(10,11)$. The results of these studies might redefine the standard treatment for NSCLC patients with EGFR mutation. Nevertheless, sequential afatinib and osimertinib treatment continues to be an important treatment option in some countries where osimertinib or RELAY regimen are exempt from insurance reimbursement.

\section{Acknowledgments}

Funding: None.

\section{Footnote}

Provenance and Peer Review: This article was a standard submission to the journal. The article has undergone external peer review.

Peer Review File: Available at https://jtd.amegroups.com/ article/view/10.21037/jtd-21-1700/prf
Conflicts of Interest: All authors have completed the ICMJE uniform disclosure form (available at https://jtd.amegroups. com/article/view/10.21037/jtd-21-1700/coif). The authors have no conflicts of interest to declare.

Ethical Statement: The authors are accountable for all aspects of the work in ensuring that questions related to the accuracy or integrity of any part of the work are appropriately investigated and resolved.

Open Access Statement: This is an Open Access article distributed in accordance with the Creative Commons Attribution-NonCommercial-NoDerivs 4.0 International License (CC BY-NC-ND 4.0), which permits the noncommercial replication and distribution of the article with the strict proviso that no changes or edits are made and the original work is properly cited (including links to both the formal publication through the relevant DOI and the license). See: https://creativecommons.org/licenses/by-nc-nd/4.0/.

\section{References}

1. Popat S, Jung HA, Lee SY, et al. Sequential afatinib and osimertinib in patients with EGFR mutation-positive NSCLC and acquired T790M: A global non-interventional study (UpSwinG). Lung Cancer 2021;162:9-15.

2. Hochmair MJ, Morabito A, Hao D, et al. Sequential afatinib and osimertinib in patients with EGFR mutationpositive non-small-cell lung cancer: final analysis of the GioTag study. Future Oncol 2020;16:2799-808.

3. Ramalingam SS, Vansteenkiste J, Planchard D, et al. Overall Survival with Osimertinib in Untreated, EGFR-Mutated Advanced NSCLC. N Engl J Med 2020;382:41-50.

4. Nosaki K, Satouchi M, Kurata T, et al. Re-biopsy status among non-small cell lung cancer patients in Japan: A retrospective study. Lung Cancer 2016;101:1-8.

5. Matsuo N, Azuma K, Sakai K, et al. Association of EGFR Exon 19 Deletion and EGFR-TKI Treatment Duration with Frequency of T790M Mutation in EGFR-Mutant Lung Cancer Patients. Sci Rep 2016;6:36458.

6. Nakagawa K, Garon EB, Seto T, et al. Ramucirumab plus erlotinib in patients with untreated, EGFRmutated, advanced non-small-cell lung cancer (RELAY): a randomised, double-blind, placebo-controlled, phase 3 trial. Lancet Oncol 2019;20:1655-69.

7. Yamaoka T, Kim YH, Iwatsubo S, et al. The Role of Dual Inhibition of EGFR and Vascular Endothelial Growth 
Factor (Receptor) in the Treatment of NSCLC With EGFR Mutation. J Thorac Oncol 2021;16:e71-2.

8. Kawashima Y, Fukuhara T, Saito H, et al. Bevacizumab plus erlotinib versus erlotinib alone in Japanese patients with advanced, metastatic, EGFR-mutant non-small-cell lung cancer (NEJ026): overall survival analysis of an openlabel, randomised, multicentre, phase 3 trial. Lancet Respir
Med 2022;10:72-82.

9. Available online: https://clinicaltrials.gov/ct2/show/ NCT04413201

10. Available online: https://clinicaltrials.gov/ct2/show/ NCT04181060

11. Available online: https://clinicaltrials.gov/ct2/show/ NCT03909334
Cite this article as: Iwamoto N, Kim YH, Iwatsubo S, Nishimura Y, Funada Y. Is there a role for sequential afatinib and osimertinib in patients with EGFR mutation? J Thorac Dis 2022;14(1):207-209. doi: 10.21037/jtd-21-1700 